\title{
Adaptive Sliding Mode Fixed-Time Tracking Control Based on Fixed-Time Sliding Mode Disturbance Observer with Dead-Zone Input
}

\author{
Hongbin Wang $\mathbb{D}$, Bo Su $(\mathbb{D}$, Yueling Wang $\mathbb{D}$, and Jing Gao \\ Lab of Industrial Computer Control Engineering of Hebei Province, Yanshan University, Qinhuangdao, China \\ Correspondence should be addressed to Bo Su; bosu@stumail.ysu.edu.cn
}

Received 25 February 2019; Revised 29 May 2019; Accepted 14 July 2019; Published 22 August 2019

Academic Editor: Basil M. Al-Hadithi

Copyright (C) 2019 Hongbin Wang et al. This is an open access article distributed under the Creative Commons Attribution License, which permits unrestricted use, distribution, and reproduction in any medium, provided the original work is properly cited.

\begin{abstract}
Aiming at the problem of fixed-time trajectory tracking control for high-order dynamic systems with external time-varying disturbance and input dead-zone, an adaptive fixed-time sliding mode control algorithm is proposed by employing a fixed-time sliding mode disturbance observer (FTSMDO) and high-order fixed-time sliding mode algorithm. Firstly, a FTSMDO is presented for the problem that estimating the compound disturbance is composed of input dead-zone and time-varying external disturbance in the higher-order dynamic system, which cannot be measured accurately. Furthermore, for the case that the total disturbance of the system has an unknown upper bound, the corresponding adaptive law is designed to estimate the unknown upper bound, and the fixed-time controller is designed based on FTSMDO algorithm to make all state variables converge in a fixed-time. Based on Lyapunov technique, the fixed-time convergence performance of the proposed algorithm is proved. The effectiveness of the presented fixed-time control algorithm is verified by simulating the depth tracking control of the underactuated underwater vehicle.
\end{abstract}

\section{Introduction}

Most of the actual physical system models have uncertainties and external disturbances, and some of them have existing dead-zone pheromone in the actuator, which greatly affects the performance of the system. Since many kinetic models of physical systems have high-order dynamic performance, especially mechanical systems, it is of great significance to study the control problems of high-order dynamic systems. It can better solve the problem of practical applications by researching on the tracking problem of high-order dynamic systems with external time-varying disturbances and actuator dead-zone. Compared with Lyapunov's asymptotically stable infinite time convergence theory, finite-time control theory pays more attention to the transient performance of the system and has practical engineering application value. With gradual maturity after several years of development, the finite-time control theory is favored by researchers because of its rapid convergence rate, higher precision, and antiinterference ability [1]. As is known that the convergence time of the finite-time algorithm is related to the initial state of the system, and with the increase of initial state, the convergence time also increases, which will greatly affect the transient performance of the system. In addition, most systems have difficulty in obtaining system initial state information, which limits the application of finite-time control theory. In contrast, the biggest advantage for the fixed-time algorithm is that there is a maximum settling time and the value is independent of the initial state. It is well applied in some mechanical systems that strictly require convergence time rate. In recent years, the fixed-time control algorithm has been further developed and achieved some results on the basis of the theory of homogeneous in [2-4], terminal sliding mode algorithm $[5,6]$, and adding power integrator algorithm $[7,8]$. It has been put into good use to deal with the fixed-time consistency of multiagents, such as the first-order multiagent system [9], the second-order multiagent system [10], and the high-order multiagent system [11]. The fixedtime control algorithm for the double-integration system has been studied in [5]. Subsequently, a novel fixed-time continuous control law for a chain of integrators of an arbitrary dimension in [2] was proposed and the convergence time 
was figured out by use of the homogeneous theory, which provided theoretical guarantee for the fixed-time control of high-order system.

Sliding mode control has good adaptability and strong robustness to system parameter perturbation and external disturbance and has fast response and insensitivity to parameter changes in sliding mode. In terms of the obvious advantages compared with other nonlinear control methods, such as small calculations and strong engineering adaptability, it has been widely used in chaotic systems, robot systems, power systems, etc. [12-14]. However, sliding mode control has a chattering phenomenon on account of its own characteristics, which makes the application range of the method suppressed. The generation of high-order sliding mode algorithm can suppress or reduce the chattering phenomenon effectively brought by sliding mode control and obtained a further study.

The universal phenomenon existing of actuator deadzone not only reduces the dynamic performance of the system, but also interferes with the stability and control accuracy of the closed-loop system, which has become one of the key issues affecting the stable operation of the system. Aiming at the problem of actuator dead-zone, scholars had carried out a lot of research work, and there have many control methods for handling nonlinear dead-zone. Whether it was for a typical second-order integral system or multiagent system, there were certain research results. The work in [15] was concerned with the problem of global robust finite-time stabilization of a class of unknown pure feedback systems with input deadzone nonlinearities by adopting the finite-time backstepping control approach. Based on the recursive method, the paper in [16] designed a new decentralized finite/fixed-time controller subject to the dead-zone input and unmodeled dynamics. Reference [7] focused on the fixed-time almost disturbance decoupling problem of nonlinear time-varying systems with multiple disturbances and dead-zone input. The problem of prescribed performance distributed output consensus for higher-order nonaffine nonlinear multiagent systems with unknown dead-zone input was investigated on account of Lyapunov stability theory and the dynamic surface control technique in [17].

For the unknown external disturbances, the best known processing methods used are fuzzy logic control, neural network control, and disturbance observer. These advanced control algorithms are used to approximate the system's uncertainty and external disturbances. As the disturbance observer has strong robustness by comparison to other control algorithms and has the advantages in small computation and easy implementation, it has been gradually developed in the past few years with the preference by more scholars. Nonlinear disturbance observers [18-20], fuzzy disturbance observer [21], sliding mode disturbance observers [22, 23], and neural network disturbance observers [24] have been proposed. For a class of nonlinear control systems with uncertainties and disturbances, a new controller which was composed of fuzzy sliding mode controller and a fuzzy based compensator has been presented in [25]. Further in order to adjust the ability of adaptively, an adaptive fuzzy structure was adopted to combine the above two methods.
It is well known that the sliding mode disturbance observer does not depend on the mathematical model of the system and is only related to the boundary of the disturbance. Nevertheless, the boundary of uncertainty in the design process is assumed to be known, but the interference in the most of systems is difficult to obtain the known boundary accurately, which sets the limitation on the application range of the disturbance observer. Besides, the observation errors of the disturbance observers in the above literatures are asymptotically convergent, and the interference can be estimated in an infinite time. On the strength of this, the works in [2628] designed a nonlinear finite-time disturbance observer for the external composite disturbance, which ensured that the interference error is finite-time convergence and improved the convergence precision. As is widely known that, the finitetime convergence observer relies heavily on the initial state of the system. In order to get rid of the dependence of the convergence time of the system on the initial state and improve the convergence rapid, fixed-time observers have been proposed and further applied in literatures [27, 29]. Unfortunately, in the above research results, with the assumption that the upper bound of disturbance is known, the biggest superiority of super-twisting algorithm is that it can effectively solve the chattering problem of the control system and enable the system to converge in a limited time and only require the derivative of the disturbance to be bounded [30]. It can be seen that the disturbance observer designed based on the fixed-time convergent super twisting algorithm also possesses the advantages mentioned above. To sum up, the high-order fixed-time sliding mode algorithm combined with the disturbance observer can further improve the robustness. It is clear that our study is motivated by the rare research of the fixed-time control algorithm for the tracking problem of high-order system with dead-zone characteristics.

This paper is formulated by the fixed-time sliding mode control of high-order dynamic system subject to external disturbance and input dead-zone. A new fixed-time sliding mode disturbance observer (FTSMDO) is proposed to estimate the complex disturbances, which is composed of external time-varying disturbance and dead-zone nonlinearities in high-order dynamic systems. The control objective is achieved when the proposed comprehensive FTSMDO with fixed-time sliding mode method can track a desired trajectory under the presence of integrated effect of input dead-zone and unknown time-varying external disturbance. Furthermore, the designed algorithm is used to applying the vertical underwater vehicle for the purpose of illustrating its effectiveness. The main contributions of this paper are as follows:

(1) A new FTSMDO based on fixed-time super-twisting algorithm is presented, which only needs the derivate time of upper boundary of disturbance. The proposed method not only relaxes the boundary conditions of the perturbation item, but also guarantees the convergence time independent of initial state. The proposed observer has simple form and small computation.

(2) Combined with the fixed-time high-order sliding mode controller, in order to make the system's state can converge equilibrium point in fixed-time under the condition 
of existing external compound disturbance, the convergence time can be figured out.

(3) On behalf of solving the problem that the upper limit of perturbation is unknown but bounded, an adaptive law is designed for adapt gain to compensate the observation error.

The essay is organized as follows. Problem formulation and preliminaries are introduced in Section 2. The adaptive fixed-time sliding mode controller is presented by composed of a new fixed-time sliding disturbance observer, fixed-time high-order sliding mode controller, and an adapt law in Section 3. Section 4 applies the developed technique to fixedtime stabilization for a 3-dof AUV control in vertical plane. Section 5 concludes this paper.

\section{Problem Statement and Preliminaries}

2.1. Problem Statement. Consider the following nonlinear integral chain system:

$$
\begin{gathered}
\dot{x}_{1}=x_{2} \\
\dot{x}_{2}=x_{3} \\
\vdots \\
\dot{x}_{n}=g(x, t) u(t)+f(x, t)+d(t)
\end{gathered}
$$

where $x_{1}, x_{2}, \cdots, x_{n}$ represent system states, $u(t) \in R$ is the control input, $f(x, t)$ is time-varying function, and $d(t)$ is the system's disturbance. Assuming that $d(t)$ is unknown, its derivative is satisfying the condition of $|\dot{d}(t)| \leq L_{1}$.

Define $x_{d}=\left(x_{1 d}, \cdots, x_{n d}\right)^{\mathrm{T}}$ is the reference value of the state $x=\left(x_{1}, \cdots, x_{n}\right)^{\mathrm{T}}$, and satisfy the relation as follows:

$$
\begin{gathered}
\dot{x}_{1 d}=x_{2 d} \\
\dot{x}_{2 d}=x_{3 d} \\
\vdots \\
\dot{x}_{(n-1) d}=x_{n d} \\
\dot{x}_{n d}=x_{1 d}^{n}
\end{gathered}
$$

Thus, the state error variable is defined by

$$
e_{1}=x_{1}-x_{1 d}, e_{2}=x_{2}-x_{2 d}, \ldots, e_{n}=x_{n}-x_{n d}
$$

The error dynamic equation can be described as

$$
\begin{gathered}
\dot{e}_{1}=e_{2} \\
\dot{e}_{2}=e_{3} \\
\vdots \\
\dot{e}_{n}=g(t) u+f\left(t, x_{1} \cdots x_{n}\right)+d(t)-\dot{x}_{n d}
\end{gathered}
$$

Consider actuator dead-zone characteristics that exist in most physical systems; the control input can be described as

$$
u=D Z(v(t))
$$

where $v(t)$ is the input signal of dead-zone and $D Z(v(t))$ denotes a dead-zone operator. The dead-zone nonlinearity of the actuator can be written by mathematic formula as follows [31]:

$$
\begin{aligned}
u(t)= & D Z(v(t)) \\
= & \begin{cases}m\left(v(t)-b_{r}\right), & \text { for } v(t) \geq b_{r} \\
0, & \text { for } b_{l}<v(t)<b_{r} \\
m\left(v(t)-b_{l}\right), & \text { for } v(t) \leq b_{l}\end{cases}
\end{aligned}
$$

where $m>0, b_{r}>0$, and $b_{l}<0$ are the dead-zone parameters. In order to provide convenience for controller design, the above dead-zone model (6) can be replaced by $D Z(v(t))=$ $m v(t)+h(v(t))$, and $h(v(t))$ is given as

$$
h(v(t))= \begin{cases}-m b_{r}, & v(t) \geq b_{r} \\ m v(t), & b_{l}<v(t)<b_{r} \\ -m b_{l}, & v(t) \leq b_{l}\end{cases}
$$

and we assume $|\dot{h}(v(t))| \leq h_{m}$, and $h_{m}$ is an unknown positive constant. For all parameters of input dead-zone, they are partially known. Namely, the parameter $m$ is known and the parameters $b_{r}$ and $b_{l}$ are unknown.

Consider the input dead-zone for system (4); the last equation can be written as follows:

$$
\dot{e}_{n}=g(x) m v(t)+f(x)+d+g h(v(t))-\dot{x}_{n d}
$$

Define the lumped disturbance as $D=g h(v(t))+d$; the derivative time of $D$ is bounded and $|\dot{D}| \leq \phi$, and $\phi$ is an unknown constant but bounded. The compound disturbance $D$ of this system contains input dead-zone and unknown external time-varying disturbance, where the derivative time of $d$ and $h$ is unknown but bounded, and we can ensure that the derivative time of $D$ exists.

Considering the dead-zone input which is described as formula (6), error equation (4), (8), and the dynamic system (1) can be rewritten into the following form:

$$
\begin{gathered}
\dot{e}_{1}=e_{2} \\
\dot{e}_{2}=e_{3} \\
\vdots \\
\dot{e}_{n}=g(x) m v(t)+f(x)+D-\dot{x}_{n d}
\end{gathered}
$$

2.2. Control Objective. The control objective is fulfilled by developing control laws $u$ such that the state of system (1) can track a reference signal in a fixed-time in the presence of dead-zone input and external disturbance. Based on the formulated tracking error system (9), the tracking problem established in this work can be reformulated as designing a method for designing a control input $v$ such that the errors $e_{i}(i=1,2 \cdots, n$. $)$ are sufficiently closing to zero in the meaning of fixed-time stability. 
2.3. Preliminaries. In the following, the main definitions and theorems used in this paper with regard to the fixed-time stability are listed.

Definition 1 (see [32]). The origin is said to be globally fixedtime stable if it is globally uniformly finite-time stable and the convergence time $T$ is globally bounded, i.e., $\exists T_{\max } \in R_{+}$ such that $T\left(x_{0}\right) \leq T_{\max }, \forall x_{0} \in R^{n}$. Therefore, in fixed-time control structures, the convergence time is always bounded independent of any initial conditions of the states.

Definition 2. The sign function can be defined as $\operatorname{sign}(x)=1$ for $x>0$; $\operatorname{sign}(x)=[1,-1]$ for $x=0$; $\operatorname{sign}(x)=-1$ for $x<0$.

Lemma 3 (see [33]). Consider the following system:

$$
\dot{x}(t)=u(t)+f(t)
$$

Here $x(t) \in R$ is system state, $u(t)$ is a control input, $f(t)$ is a disturbance satisfying Lipchitz condition with a certain constant $L$, and $|\dot{f}(x, t)| \leq L$.

Consider the following control input:

$$
\begin{aligned}
u(t)= & -\phi_{1} \operatorname{sig}(x(t))^{1 / 2}-\phi_{2} \operatorname{sig}(x(t))^{p} \\
& -\eta \int_{t_{0}}^{t} \operatorname{sign}(x(t)) \mathrm{d} s
\end{aligned}
$$

Here, $\phi_{1}, \phi_{2}, \eta>0, p>1, \operatorname{sig}(\cdot)^{\chi}=|\cdot|^{\chi} \operatorname{sign}(\cdot)$, the resulting closed-loop system (10) is fixed-time convergent to the origin, and the convergence is

$$
\begin{aligned}
T_{1} \leq & \left(\frac{1}{\phi_{2}(p-1) \varepsilon^{p-1}}+\frac{2 \varepsilon^{1 / 2}}{\phi_{1}}\right) \\
& \cdot\left(1+\frac{1}{m\left(1 / M-h\left(\phi_{1}\right) / \phi_{1}\right)}\right)
\end{aligned}
$$

where $\varepsilon>0, M=\eta+L, m=\eta-L, h\left(\phi_{1}\right)=1 / \phi_{1}+$ $\left(2 e / m \phi_{1}\right)^{1 / 3}$, and $e$ is the base of the natural logarithms, provided that the following conditions hold for control gains: $\eta>L, \phi_{1} h^{-1}\left(\phi_{1}\right)>M$. The minimum value of $T_{1}$ is reached for $\varepsilon=\left(\phi_{1} / \phi_{2}\right)^{1 /(p+1 / 2)}$. Specially, in a vector case $x(t) \in R^{n}, a$ continuous control law supplying global fixed-time convergence to the origin for system (10)

$$
\begin{aligned}
u(t)= & -\phi_{1} \frac{x(t)}{\|x(t)\|^{1 / 2}}-\phi_{2} x(t)\|x(t)\|^{p-1} \\
& -\eta \int_{t_{0}}^{t} \frac{x(s)}{\|x(s)\|} \mathrm{d} s
\end{aligned}
$$

Lemma 4 (see [2]). Consider the following system:

$$
\begin{gathered}
\dot{x}_{1}=x_{2} \\
\dot{x}_{2}=x_{3} \\
\ldots \\
\dot{x}_{n}=u(t)
\end{gathered}
$$

The control input in system (14) can be designed as follows:

$$
u(t)=u_{1}(t)+u_{2}(t)
$$

where the control law $u_{1}(t)$ and $u_{2}(t)$ are, respectively, given as follows:

$$
\begin{aligned}
& u_{1}(t)=v_{1}(t)+v_{2}(t)+\cdots+v_{n}(t) \\
& u_{2}(t)=\omega_{1}(t)+\omega_{2}(t)+\cdots+\omega_{n}(t) \\
& v_{i}(t)=-k_{i}\left|x_{i}(t)\right|^{\gamma_{i}} \operatorname{sign}\left(x_{i}(t)\right) \\
& \omega_{i}(t)=-K_{i}\left|x_{i}(t)\right|^{\eta_{i}} \operatorname{sign}\left(x_{i}(t)\right)
\end{aligned}
$$

where $i=1, \ldots, n$. The exponents $\gamma_{i}$ and $\eta_{i}$ are selected in accordance with $\gamma_{i} \in(0,1), i=1, \ldots, n$, satisfy the recurrent relations $\gamma_{i-1}=\gamma_{i} \gamma_{i+1} /\left(2 \gamma_{i+1}-\gamma_{i}\right), i=2, \ldots, n, \gamma_{n+1}=1$ and $\gamma_{n}=\gamma ; \eta_{i}>1, i=1, \ldots, n$, satisfy the recurrent relations $\eta_{i-1}=\eta_{i} \eta_{i+1} /\left(2 \eta_{i+1}-\eta_{i}\right), i=2, \ldots, n, \eta_{n+1}=1$ and $\eta_{n}=\eta$, where $\gamma$ belongs to an interval $(1-\varepsilon, 1)$ and $\eta$ belongs to an interval $\left(1,1+\varepsilon_{1}\right)$, for sufficiently small $\varepsilon>0$ and $\varepsilon_{1}>0$. Control gains $k_{i}$ and $K_{i}, i=1, \ldots, n$, are assigned such that $s^{n}+k_{n} s^{n-1}+\cdots+k_{1}$ and $s^{n}+K_{n} s^{n-1}+\cdots+K_{1}$ are Hurwitz polynomials. Additionally, the following matrices are used to meet the condition of Hurwitz

$$
\begin{aligned}
A & =\left(\begin{array}{ccccc}
0 & 1 & 0 & \cdots & 0 \\
0 & 0 & 1 & \cdots & 0 \\
\cdots & & & & \\
0 & 0 & 0 & \cdots & 1 \\
-k_{1} & -k_{2} & -k_{3} & \cdots & -k_{n}
\end{array}\right) \\
A_{1} & =\left(\begin{array}{ccccc}
0 & 1 & 0 & \cdots & 0 \\
0 & 0 & 1 & \cdots & 0 \\
\cdots & & & & \\
0 & 0 & 0 & \cdots & 1 \\
-K_{1} & -K_{2} & -K_{3} & \cdots & -K_{n}
\end{array}\right)
\end{aligned}
$$

The convergence time of the above approach is $T_{2}$, which satisfies the inequality:

$$
T_{2} \leq \frac{\lambda_{\text {max }}^{\rho}(P)}{r_{0} \rho}+\frac{1}{r_{1} \omega r^{\omega}}
$$

where $\rho=(1-\gamma) / \gamma, \omega=(\eta-1) / \eta, r_{0}=\lambda_{\text {min }}(Q) / \lambda_{\text {max }}(P), r_{1}=$ $\lambda_{\text {min }}\left(Q_{1}\right) / \lambda_{\text {max }}\left(P_{1}\right)$, and $r \leq \lambda_{\text {min }}\left(P_{1}\right)$ is a positive number. $Q, Q_{1}, P$, and $P_{1}$ are symmetric positive definite matrices and satisfy the following equation relations $P A+A^{T} P=-Q$, $P_{1} A_{1}+A_{1}{ }^{T} P_{1}=-Q_{1} \cdot \lambda_{\text {min }}(\cdot)$ and $\lambda_{\text {max }}(\cdot)$ are defined as the maximum and minimum eigenvalues of the matrix, respectively. 


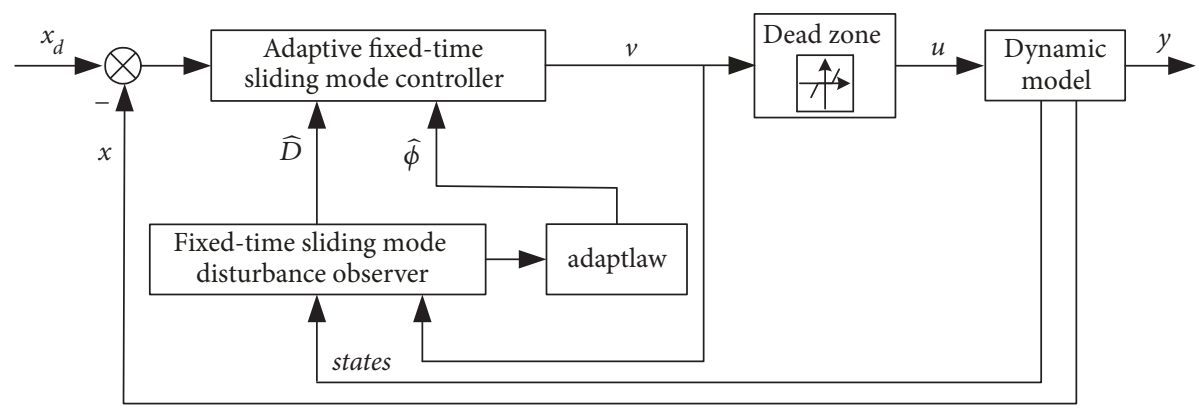

FIgURE 1: The diagram of controller's block.

Upon substituting the control input (16) into (14), the resulting fixed-time convergence system (14), we have

$$
\begin{aligned}
& \dot{x}_{1}(t)=x_{2}(t), x_{1}\left(t_{0}\right)=x_{10}, \dot{x}_{2}(t)=x_{3}(t), x_{2}\left(t_{0}\right) \\
& \quad=x_{20} \\
& \quad \cdots \\
& \dot{x}_{n}(t)=v_{1}(t)+v_{2}(t)+\cdots+v_{n}(t)+\omega_{1}(t)+\omega_{2}(t) \\
& \quad+\cdots+\omega_{n}(t), x_{n}\left(t_{0}\right)=x_{n 0}
\end{aligned}
$$

\section{Controller Design}

In this section, an adaptive fixed-time sliding mode controller in conjunction with a new fixed-time sliding mode disturbance observer (FTSMDO) will be proposed to make all states of the system (9) quite small within a fixed-time stable. First, a new fixed-time sliding mode disturbance observer is presented. Then, a fixed-time sliding mode controller conjunction with the presented disturbance observer is designed. Meanwhile, an adaptive law is adopted in dealing with the upper bounds of disturbance. The controller's block diagram designed in this paper is shown in Figure 1.

3.1. Fixed-Time Sliding Mode Disturbance Observer Based on Compound Perturbation. Sliding mode disturbance observer is often used in control system to cope with uncertainty and disturbance in order to strengthen robustness. However, existing SMDO method is asymptotically or finite-time convergence; in other words, the convergence time is related to the initial state and prolonged. More importantly, chattering phenomenon is the shortcoming of the SMDO. Thus, with the direction at the compound disturbance existing in the system (9), a new fixed-time sliding mode disturbance observer (FTSMDO) based on super-twisting structure is designed. First, the sliding surface is defined as follows:

$$
S_{0}=c_{1} e_{1}+c_{2} e_{2}+\cdots+c_{n-1} e_{n-1}+e_{n}
$$

where $S_{0} \in R$ and $c_{n}(n=1,2, \cdots n-1)$, which satisfy polynomial Hurwitz.

Taking the derivative of the surface $S_{0}$ in (20),

$$
\dot{S}_{0}=c_{1} e_{2}+c_{2} e_{3}+\cdots+c_{n-1} e_{n}+\dot{e}_{n}
$$

Theorem 5. Consider system (9) that contains external compound disturbance, then if we choose the following auxiliary variables $\sigma=S_{0}+Z, Z$ is designed auxiliary variable, then the disturbance item $D$ in the system can be estimated within a fixed-time $T_{1}$, and $\sigma$ satisfies the following equations:

$$
\begin{aligned}
& u=-\phi_{1} \operatorname{sig}(\sigma)^{1 / 2}-\phi_{2} \operatorname{sig}(\sigma)^{p}+y \\
& \dot{y}=-\eta \operatorname{sign}(\sigma)
\end{aligned}
$$

Here, $T_{1}$ satisfies inequality (12).

Proof. For system (9), selecting auxiliary variable $z$ and figuring out the derivative of $z$ yield to

$$
\dot{Z}=-c_{1} e_{2}-c_{2} e_{3}-\cdots-c_{n-1} e_{n}-f(\mathrm{x})+\dot{\mathrm{x}}_{n d}+\widetilde{u}+u_{i n}
$$

where the real control input can be written as $v(t)=$ $(g(x) M)^{-1} \tilde{u}$ and $u_{\text {in }}$ is auxiliary control input. Simultaneously, considering (21), (22), and (23), we can calculate some equations about $\sigma$.

$$
\begin{aligned}
& \sigma=S_{0}+Z \\
& \dot{\sigma}=\dot{S}_{0}+\dot{Z}=u_{i n}+D \\
& \dot{\sigma}=D+u_{i n}=D-\phi_{1} \operatorname{sig}(\sigma)^{1 / 2}-\phi_{2} \operatorname{sig}(\sigma)^{p}+y \\
& \dot{y}=-\eta \operatorname{sign}(\sigma) \\
& \dot{\sigma}=D-\widehat{D}
\end{aligned}
$$

The second-order sliding mode techniques are able to stabilize not only the sliding variable to zero, but also its first-order successive derivative. According to the rule of Lemma 3, $\sigma$ and $\dot{\sigma}$ in equation (24) can converge to zero within a fixed-time.

$$
\sigma=\dot{\sigma}=0, \quad \forall t>T_{1}
$$

Once the condition of $\dot{\sigma}=0$ is satisfied, we may get $D=$ $-u_{i n}$. Therefore, it can be seen that $-u_{\text {in }}$ can accurately estimate uncertainty variable $D$ within a fixed-time $T_{1}$ and equivalent $\widehat{D}=-u_{i n}$. Specially, for any vector $e=\left[e_{1}, e_{2} \cdots e_{n}\right]^{T} \in R^{n}$ of system (9), we have $\widehat{D}=\phi_{1}\left(\sigma(t) /\|\sigma(t)\|^{1 / 2}\right)+\phi_{2} \sigma(t)\|\sigma(t)\|^{p-1}+$ $\int_{t_{0}}^{t} \eta(\sigma(t) /\|\sigma(t)\|) d s$ according to formula (13); the details are similar to Theorem 5 we presented and are omitted here for the sake of simplifying the expression. 
Remark 6. A FTSMDO is developed to estimate the compound disturbance $\mathrm{D}$, which contains external time-varying disturbance and input dead-zone. Unlike the asymptotically convergent disturbance observer, the FTSMDO is fixed-time convergent free from the constraint of the initial state and has better anti-interference ability and rapid convergence. The restrictive condition focus on the designed is relaxed, which only requires the unknown upper boundary for the time derivative of the sum of disturbance. The convergence time of the FTSMDO is related to the controller parameters only.

Remark 7. The integral operation is implemented on the sign function existing in the introduced auxiliary control input, so $u_{\text {in }}$ is continuous, and then the control input $v$ is smooth and continuous.

3.2. Comprehensive Design of Adaptive Fixed-Time Sliding Mode Controller Based on FTSMDO. As a result of the unpredictability of external disturbances, it is difficult to determine the upper bound of the disturbance in practical engineering. In order to obtain more accurate interference values and ensure the best performance of the system, an adaptive law is designed for the upper bound of the disturbance, and a fixed-time convergence control algorithm with adaptive gain was studied to estimate the upper bound of the external interference of the model. A FTSMDO conjunction with adaptive sliding mode fixed-time controller is proposed for error dynamic system (9), which guarantees system's states can also converge in a fixed-time under the condition of unknown upper boundary for the compound disturbance, and the trajectory of the system can track the desired trajectory in a fixed-time.

In the following, we assume that the lumped disturbance satisfying the condition of $|\dot{D}|<\phi, \phi$ is unknown, and $\widehat{\phi}$ is parameter estimation for uncertain boundary. Define error as $\widetilde{\phi}=\widehat{\phi}-\phi$. Due to the unpredictability of external interference, in order to ensure the optimal control performance of the system, it is necessary to estimate interference error $\widetilde{D}$ obtained by the observer, assuming that the estimated amount is $\widehat{D}$. Here, we define $\widetilde{D}=\widehat{D}-D$.

For system (9), the sliding mode manifold is designed in the following form:

$$
\begin{aligned}
s= & \dot{e}_{n}+k_{1}\left|e_{1}\right|^{\gamma_{1}} \operatorname{sign}\left(e_{1}\right)+\cdots \\
& +k_{n-1}\left|e_{n-1}\right|^{\gamma_{n-1}} \operatorname{sign}\left(e_{n-1}\right)+k_{n}\left|e_{n}\right|^{\gamma_{n}} \operatorname{sign}\left(e_{n}\right) \\
& +K_{1}\left|e_{1}\right|^{\eta_{1}} \operatorname{sign}\left(e_{1}\right)+\cdots \\
& +K_{n-1}\left|e_{n-1}\right|^{\eta_{n-1}} \operatorname{sign}\left(e_{n-1}\right)+K_{n}\left|e_{n}\right|^{\eta_{n}} \operatorname{sign}\left(e_{n}\right)
\end{aligned}
$$

Theorem 8. For system (9), the control input is defined as

$$
v(t)=(g m)^{-1}\left(u_{e q}+u_{n}\right)
$$

With the FTSMDO (24), we consider $u_{e q}$ as follows:

$$
\begin{aligned}
u_{e q}= & -f(x)+\dot{x}_{n d}-\widehat{D}-k_{1}\left|e_{1}\right|^{\gamma_{1}} \operatorname{sign}\left(e_{1}\right) \\
& -k_{2}\left|e_{2}\right|^{\gamma_{2}} \operatorname{sign}\left(e_{2}\right) \cdots-k_{n}\left|e_{n}\right|^{\gamma_{n}} \operatorname{sign}\left(e_{n}\right) \\
& -K_{1}\left|e_{1}\right|^{\eta_{1}} \operatorname{sign}\left(e_{1}\right)-K_{2}\left|e_{2}\right|^{\eta_{2}} \operatorname{sign}\left(e_{2}\right) \cdots \\
& -K_{n}\left|e_{n}\right|^{\eta_{n}} \operatorname{sign}\left(e_{n}\right)
\end{aligned}
$$

and

$$
\dot{u}_{n}=-(\widehat{\phi}+\eta) \operatorname{sign}(s)
$$

Adaptive law is designed as

$$
\dot{\widehat{\phi}}=\frac{1}{\beta}|s|
$$

with $\eta>0$ is the gain parameter $(\eta \in R)$. For the error dynamic system (9), on basic of the sliding mode surface equation (26), if we chose control law as (27) with the FTSMDO as (24) and the adaptive law as (30), which can achieve fixed-time stabilization and provide convergence of the states in a fixedtime, the convergence time is given as $T_{z} \leq T_{1}+T_{2}$ :

$$
\begin{aligned}
T_{z} \leq & \frac{\lambda_{\max }^{\rho}(P)}{r_{0} \rho}+\frac{1}{r_{1} \omega r^{\omega}} \\
+ & \left(\frac{1}{\phi_{2}(p-1) \varepsilon^{p-1}}+\frac{2 \varepsilon_{1}^{1 / 2}}{\phi_{1}}\right) \\
& \cdot\left(1+\frac{1}{m\left(1 / M-h\left(\phi_{1}\right) / \phi_{1}\right)}\right)
\end{aligned}
$$

Proof. Substituting (27)-(29) into (26), we will get

$$
s=-\widetilde{D}+u_{n}
$$

The derivative of the above equation is derived as

$$
\dot{s}=-\dot{\widetilde{D}}+\dot{u}_{n}
$$

when $t>T_{1}$, where $T_{1}$ is the upper bound of FTSMDO convergence time, $\widetilde{D}=0$. Define Lyapunov function with respect to $s$ and $\widetilde{\phi}$ as follows:

$$
V=\frac{1}{2} s^{2}+\frac{1}{2 \kappa}(\widehat{\phi}-\phi)^{2}
$$


The time derivative of $V$ is figured out as follows:

$$
\begin{aligned}
& \dot{V}=s \dot{s}+\frac{1}{\kappa}(\widehat{\phi}-\phi) \dot{\hat{\phi}}=s\left(\dot{u}_{n}\right)+\frac{1}{\kappa \beta}(\widehat{\phi}-\phi)|s| \\
& \leq-(\eta+\widehat{\phi})|s|+\frac{1}{\kappa \beta}(\widehat{\phi}-\phi)|s|=-(\eta+\widehat{\phi})|s| \\
& +\phi|s|-\phi|s|+\frac{1}{\kappa \beta}(\widehat{\phi}-\phi)|s|=-(\widehat{\phi}-\phi)|s| \\
& -(\eta+\phi)|s|+\frac{1}{\kappa \beta}(\widehat{\phi}-\phi)|s|=-(\eta+\phi)|s| \\
& -\left(1-\frac{1}{\kappa \beta}\right)|s|(\hat{\phi}-\phi)=-(\eta+\phi) \sqrt{2} \frac{|s|}{\sqrt{2}} \\
& -\left(1-\frac{1}{\kappa \beta}\right)|s| \sqrt{2} \frac{(\hat{\phi}-\phi)}{\sqrt{2}}=-(\eta+\phi) \sqrt{2} \frac{|s|}{\sqrt{2}} \\
& -\left(1-\frac{1}{\kappa \beta}\right)|s| \sqrt{2} \frac{(\widehat{\phi}-\phi)}{\sqrt{2}} \leq-(\eta+\phi) \\
& \cdot \sqrt{2}\left(\frac{|s|^{2}}{2}\right)^{1 / 2}-\left(1-\frac{1}{\kappa \beta}\right)|s| \sqrt{2}\left(\frac{|\hat{\phi}-\phi|^{2}}{2}\right)^{1 / 2} \\
& \leq-\min \left\{(\eta+\phi) \sqrt{2},\left(1-\frac{1}{\kappa \beta}\right)|s| \sqrt{2}\right\} \\
& \left(\frac{s^{2}}{2}+\frac{(\widehat{\phi}-\phi)^{2}}{2}\right)^{1 / 2} \\
& \leq-\min \left\{(\eta+\phi) \sqrt{2},\left(1-\frac{1}{\kappa \beta}\right)|s| \sqrt{2}\right\} V^{1 / 2}
\end{aligned}
$$

where $\kappa$ and $\beta$ are positive constants satisfying the relation as $\kappa>1 / \beta$.

If the control law (27) is used for the system (9) during sliding, the state variables can converge equilibrium point in a fixed-time and yield to the following form of equations:

$$
\begin{gathered}
\dot{e}_{1}=e_{2} \\
\dot{e}_{2}=e_{3} \\
\vdots \\
\dot{e}_{n}=-k_{1}\left|e_{1}\right|^{\gamma_{1}} \operatorname{sign}\left(e_{1}\right)-k_{2}\left|e_{2}\right|^{\gamma_{2}} \operatorname{sign}\left(e_{2}\right) \cdots \\
\quad-k_{n}\left|e_{n}\right|^{\gamma_{n}} \operatorname{sign}\left(e_{n}\right)-K_{1}\left|e_{1}\right|^{\eta_{1}} \operatorname{sign}\left(e_{1}\right) \\
-K_{2}\left|e_{2}\right|^{\eta_{2}} \operatorname{sign}\left(e_{2}\right) \cdots-K_{n}\left|e_{n}\right|^{\eta_{n}} \operatorname{sign}\left(e_{n}\right)
\end{gathered}
$$

The above-mentioned system is fixed-time stable in accordance with the Lemma 4, which means that the sliding mode surface will reach $s=0$ in fixed-time and then along $s=0$ within a max settling time $T_{2}$, where $T_{2}$ is the maximum convergence time estimated by inequality (18). Considering the fact that when $t>T_{1}$ and according to Theorem 5,
$\widetilde{D}$ converges to the origin in fixed-time $T_{1}$ which can be calculated by inequality (12). So the total convergence time considering the convergence of FTSMDO is $T_{z} \leq T_{1}+T_{2}$.

It is demonstrated that formula (35) enables the sliding mode $s(t)$ to converge to zero in finite time. Substituted controller (27) into system (9) and obtained equivalent to system (14). According to Lemma 4, system (9) can converge in fixed-time under the design of adaptive fixed-time controller (27), sliding mode fixed-time disturbance observer (24), and adaptive law (30).

Remark 9. In the high-order dynamic system subject to deadzone and external disturbance, an adaptive fixed-time sliding mode controller based on FTSMDO is proposed. More importantly, the switching term of the disturbance observer is on the high-order sliding mode surface. While chattering phenomenon of the system being avoided, the selection of the sliding mode surface at a fixed-time also avoids the chattering phenomenon brought by the sliding mode algorithm; thus the control input of the application system is not destroyed or weakened. In summary, practical significance and theoretical challenges are achieved by the fixed-time theoretical research on the tracking control problem of high-order dynamic systems with external time-varying disturbances and input dead-zones.

Remark 10. In some actual situation, the successive derivative of the reference signal is not easy available directly by use of derivative approach. In order to acquire the high-order derivative of the reference value, a fixed-time differentiator can be borrowed [34].

$$
\begin{gathered}
\dot{\xi}_{1}=\xi_{2}-k_{1}\left|\xi_{1}-y(t)\right|^{a_{1}} \operatorname{sign}\left(\left|\xi_{1}-y(t)\right|\right) \\
-\kappa_{1}\left|\xi_{1}-y(t)\right|^{\beta_{1}} \operatorname{sign}\left(\left|\xi_{1}-y(t)\right|\right) \\
\vdots \\
\dot{\xi}_{i}=\xi_{i+1}-k_{i}\left|\xi_{1}-y(t)\right|^{a_{i}} \operatorname{sign}\left(\left|\xi_{1}-y(t)\right|\right) \\
-\kappa_{i}\left|\xi_{1}-y(t)\right|^{\beta_{i}} \operatorname{sign}\left(\left|\xi_{1}-y(t)\right|\right) \\
\vdots \\
\dot{\xi}_{n}=-k_{n}\left|\xi_{1}-y(t)\right|^{a_{n}} \operatorname{sign}\left(\left|\xi_{1}-y(t)\right|\right) \\
-\kappa_{n}\left|\xi_{1}-y(t)\right|^{\beta_{n}} \operatorname{sign}\left(\left|\xi_{1}-y(t)\right|\right)
\end{gathered}
$$

where $\xi_{1}$ is the estimated value of $x_{1 d}, \xi_{2}$ present the derivative of $\dot{x}_{1 d}$, and correspondingly, $\xi_{n}$ is the derivative of $x_{1 d}^{(n-1)}$. Moreover, the system parameter value can be figured according to the Theorem 2 in [34].

The next section presents application of the designed fixed-time convergent control law to control an underactuated AUV in vertical plane. 


\section{Research on Fixed-Time Control for the Vertical Plane Control of Autonomous Underwater Vehicle}

The vertical plane of an underactuated AUV is simplified as follows $[35,36]$ :

$$
\begin{aligned}
\left(I_{\mathrm{yy}}-M_{\dot{q}}\right) \dot{q} & =M_{u q} u q-z_{G} W \theta+M_{u u} u^{2} \delta_{s}+d_{t u r} \\
\dot{\theta} & =q \\
\dot{z} & =-h \theta
\end{aligned}
$$

where $q$ is the pitch angle velocity, $\theta$ is the pitch angle, $z$ is the depth of AUV, and $h$ is the velocity in the surge direction. $\delta_{s}$ denotes the rudder input, $d_{t u r}$ is the disturbance imposed in the pitch motion, and the rest of the parameters of the above equation are hydrodynamics parameters and not to introduce too much here. Assumed that the state quantity of (38) can be measured, the above equation can be written into a short form as follows:

$$
\begin{aligned}
& \dot{\mathrm{z}}=-h \theta \\
& \dot{\theta}=q \\
& \dot{q}=M_{1} q+M_{2} \theta+M_{3} \delta_{s}+d_{0}
\end{aligned}
$$

The coefficients $M_{i}(i=1,2,3)$ and interference parameter $d_{0}$ are shown as

$$
\begin{aligned}
& M_{1}=\frac{M_{u q} u}{I_{y y}-M_{\dot{q}}}, \\
& M_{2}=\frac{M_{\theta}}{I_{y y}-M_{\dot{q}}}, \\
& M_{3}=\frac{M_{u u} h^{2}}{I_{y y}-M_{\dot{q}}}, \\
& M_{\theta}=-z_{G} W, \\
& d_{0}=\frac{d_{t u r}}{I_{y y}-M_{\dot{q}}}
\end{aligned}
$$

For research needs convenient, take state variable replacement $\dot{z}=w$; system (39) is converted into the fellow form in regard to the state new variables $z, w, p$ :

$$
\begin{aligned}
\dot{z} & =w \\
\dot{w} & =p \\
\dot{p} & =M_{1} p+M_{2} w-h M_{3} \delta_{s}+d
\end{aligned}
$$

where $d=h d_{0}$. Considering the dead-zone phenomenon existing in the process of underwater robot dive control, here we select $\delta_{s}$ equivalent to $u(t)$ of (7).

In the light of the first and second equation in (39), the state variables $w$ and $p$ are available. The third equation in (41) contains disturbance and does not obtain directly, so we adopt FTSMDO to estimate it; thus the state variable $p$ is available. Considering the depth tracking desired value $z_{d}$, the error equation of (42) could be rewritten as

$$
\begin{aligned}
& \dot{e}_{1}=e_{2} \\
& \dot{e}_{2}=e_{3} \\
& \dot{e}_{3}=M_{1} p+M_{2} w-\dddot{z}_{d}-h M_{3} v(t)+D
\end{aligned}
$$

The true control input can be written as follows:

$$
v=-\left(h M_{3} m\right)^{-1}\left(u_{e q}+u_{n}\right)
$$

where

$$
\begin{aligned}
u_{e q}= & -\left(M_{1} p+M_{2} w\right)+\dddot{z}_{d}-k_{1}\left|e_{1}\right|^{\gamma_{1}} \operatorname{sign}\left(e_{1}\right) \\
& -k_{2}\left|e_{2}\right|^{\gamma_{2}} \operatorname{sign}\left(e_{2}\right)-k_{3}\left|e_{3}\right|^{\gamma_{3}} \operatorname{sign}\left(e_{3}\right) \\
& -K_{1}\left|e_{1}\right|^{\eta_{1}} \operatorname{sign}\left(e_{1}\right)-K_{2}\left|e_{2}\right|^{\eta_{2}} \operatorname{sign}\left(e_{2}\right) \\
& -K_{3}\left|e_{3}\right|^{\eta_{3}} \operatorname{sign}\left(e_{3}\right)-\widehat{D}
\end{aligned}
$$

and $\dot{u}_{n}=-(\widehat{\phi}+\eta) \operatorname{sign}(s)$.

According to the control algorithm (26) proposed in this paper, design the following controller:

$$
\begin{aligned}
s= & \dot{e}_{3}+k_{1}\left|e_{1}\right|^{\gamma_{1}} \operatorname{sign}\left(e_{1}\right)+k_{2}\left|e_{2}\right|^{\gamma_{2}} \operatorname{sign}\left(e_{2}\right) \\
& +k_{3}\left|e_{3}\right|^{\gamma_{3}} \operatorname{sign}\left(e_{3}\right)+K_{1}\left|e_{1}\right|^{\eta_{1}} \operatorname{sign}\left(e_{1}\right) \\
& +K_{2}\left|e_{2}\right|^{\eta_{2}} \operatorname{sign}\left(e_{2}\right)+K_{3}\left|e_{3}\right|^{\eta_{3}} \operatorname{sign}\left(e_{3}\right)
\end{aligned}
$$

where, in accordance with the Theorem 5 and (7)-(12), the sliding mode surface is selected and auxiliary variable calculated leave each other as follows:

The sliding surface $S_{0}$ is designed as follows:

$$
S_{0}=c_{1} e_{1}+c_{2} e_{2}+e_{3}
$$

The time derivative of above equation is

$$
\dot{S}_{0}=c_{1} e_{2}+c_{2} e_{3}+\dot{e}_{3}
$$

The auxiliary variable $Z$ is assumed

$$
\sigma=S_{0}+Z
$$

The derivatives of $S$ and $Z$ are calculated, respectively, as follows:

$$
\begin{aligned}
& \dot{Z}=-c_{1} e_{2}-c_{2} e_{3}-M_{1} p-M_{2} w+h M_{3} m v+\dddot{z}_{d}+u_{i n} \\
& \dot{\sigma}=\dot{S}_{0}+\dot{Z}=u_{i n}+D
\end{aligned}
$$

As mentioned above, the disturbance observer is designed as follows:

$$
\begin{aligned}
u_{\text {in }} & =-\phi_{1} \operatorname{sig}(\sigma)^{1 / 2}-\phi_{2} \operatorname{sig}(\sigma)^{p}+y \\
\dot{y} & =-\eta \operatorname{sign}(\sigma) \\
\widehat{D} & =-u_{\text {in }}
\end{aligned}
$$



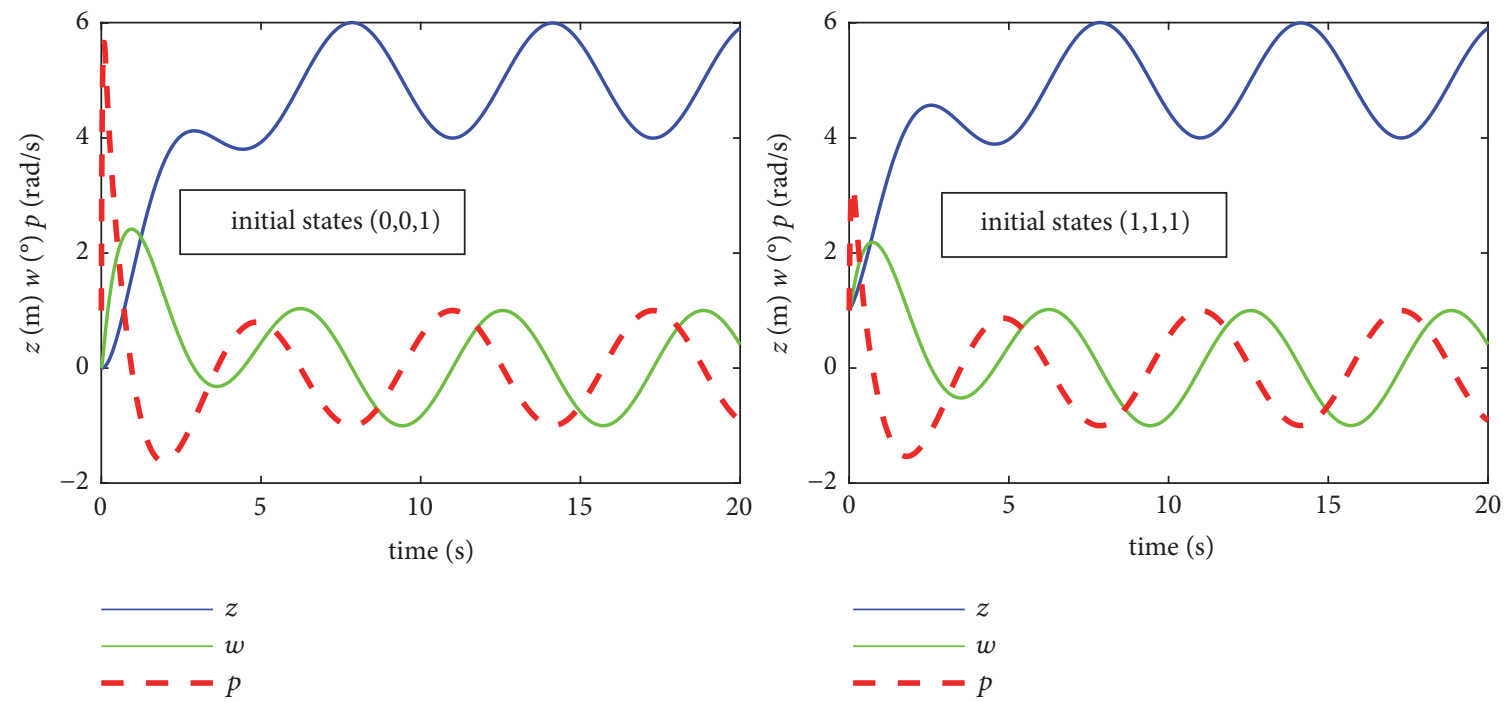

FIgURE 2: The AUV vertical plane system states $[z, w, p]$ in the entire simulation interval for the case of initial states as [001] and [111], respectively.
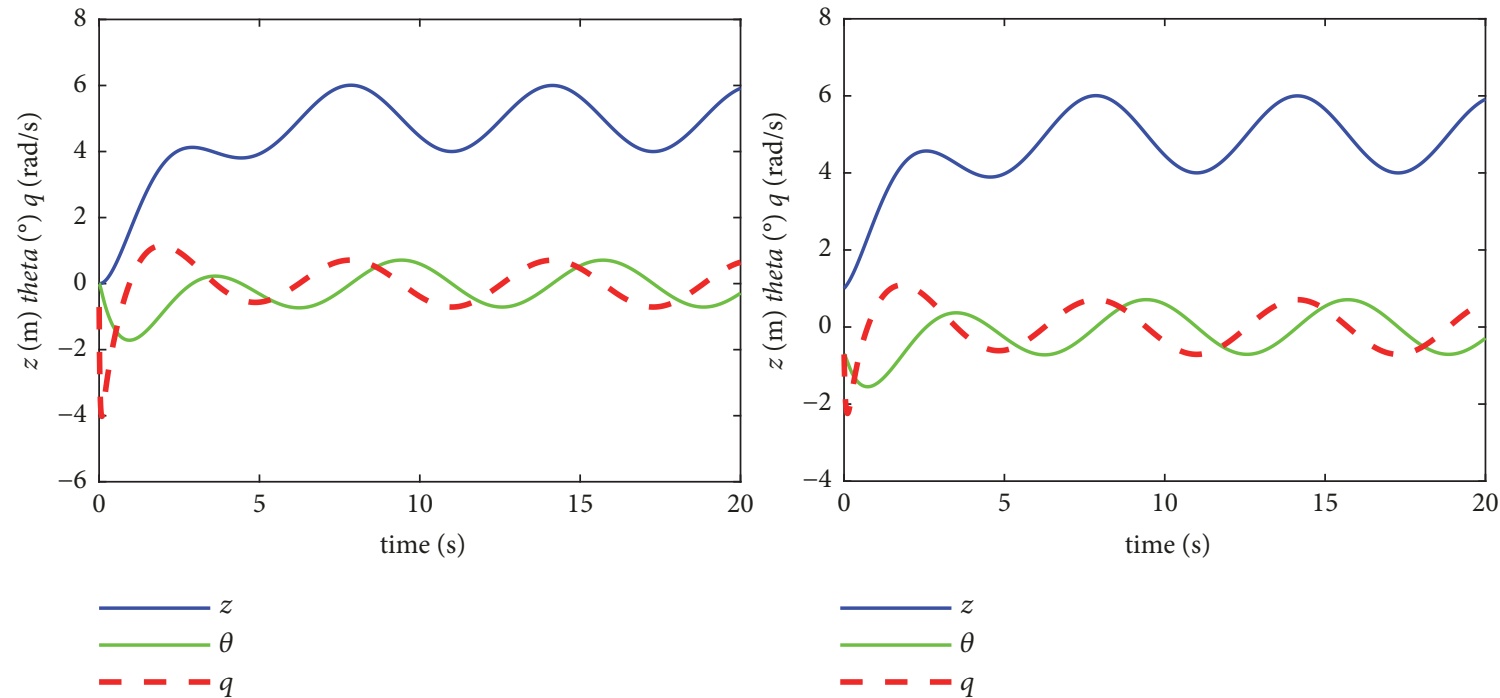

FIGURE 3: The AUV vertical plane system states $[z, \theta, q]$ in the entire simulation interval.

Here, the exponents of (45) are selected as $\gamma_{1}=19 / 20$, $\gamma_{2}=19 / 21, \gamma_{3}=19 / 22, \eta_{1}=21 / 18, \eta_{2}=21 / 19$, and $\eta_{3}=21 / 20$; the control gains are set to $k_{1}=K_{1}=6$, $k_{2}=K_{2}=11$, and $k_{3}=K_{3}=6$. After trial and error, the FTSMDO parameters are set to $c_{1}=1, c_{2}=2, \phi_{1}=4, \phi_{2}=8$, $\eta=6$, and $p=1.5$. We selected the disturbance as $d_{0}=$ $1000 \sin (t)$ and the tracking trajectory as $z_{d}=5+\sin (t)$. The parameter values of the underactuated underwater vehicle used for simulation are $I_{y y}=119.1 \mathrm{~kg} \cdot \mathrm{m}^{2}, M_{u u}=-118.56 \mathrm{~kg}$. $\mathrm{s}^{2} \cdot \mathrm{rad}^{-1}, M_{\dot{q}}=-93.3 \mathrm{~kg} \cdot \mathrm{m}^{2} \cdot \mathrm{rad}^{-1}, M_{\theta}=-28.3 \mathrm{~kg} \cdot \mathrm{m}^{2} \cdot \mathrm{rad}^{-1}$, $M_{u q}=-1008.7 \mathrm{~kg} \cdot \mathrm{s} \cdot \mathrm{rad}^{-1}, g=9.81 \mathrm{~kg} \cdot \mathrm{m} \cdot \mathrm{s}^{-1}, u=$ $1.41 \mathrm{~m} \cdot \mathrm{s}^{-1}, m=222 \mathrm{~kg}$.

In order to illustrate the fixed-time tracking performance of the designed control algorithm, the initial states are set to two situations which are $[0 ; 0 ; 1]$ and $[1 ; 1 ; 1]$ in Figures $2-7$. We compared the responses of two different initial values of the AUV system. The simulation graphs for state variables $[z(t) ; w(t) ; p(t)]$ corresponding to the initial values $[0 ; 0 ; 1]$ and $[1 ; 1 ; 1]$ are given, respectively, in Figure 2 . The simulation graphs for the original variables $[z(t) ; \theta(t) ; q(t)]$ are presented in Figure 3. Figure 4 shows the disturbance $d$ and its estimate $\widehat{d}$ without consideration dead-zone in the entire simulation interval $[0,20]$, and the curve is given to better show the dynamic changes. Figure 5 demonstrates the control input law $\delta_{s}$ against the disturbance $\widehat{d}$. Figure 6 shows a comparison of observers with two initial states situation. Figure 7 shows the adapt law curve in different initial states. From the simulation results, we can see that, as the initial values changed, the convergence time of AUV is 

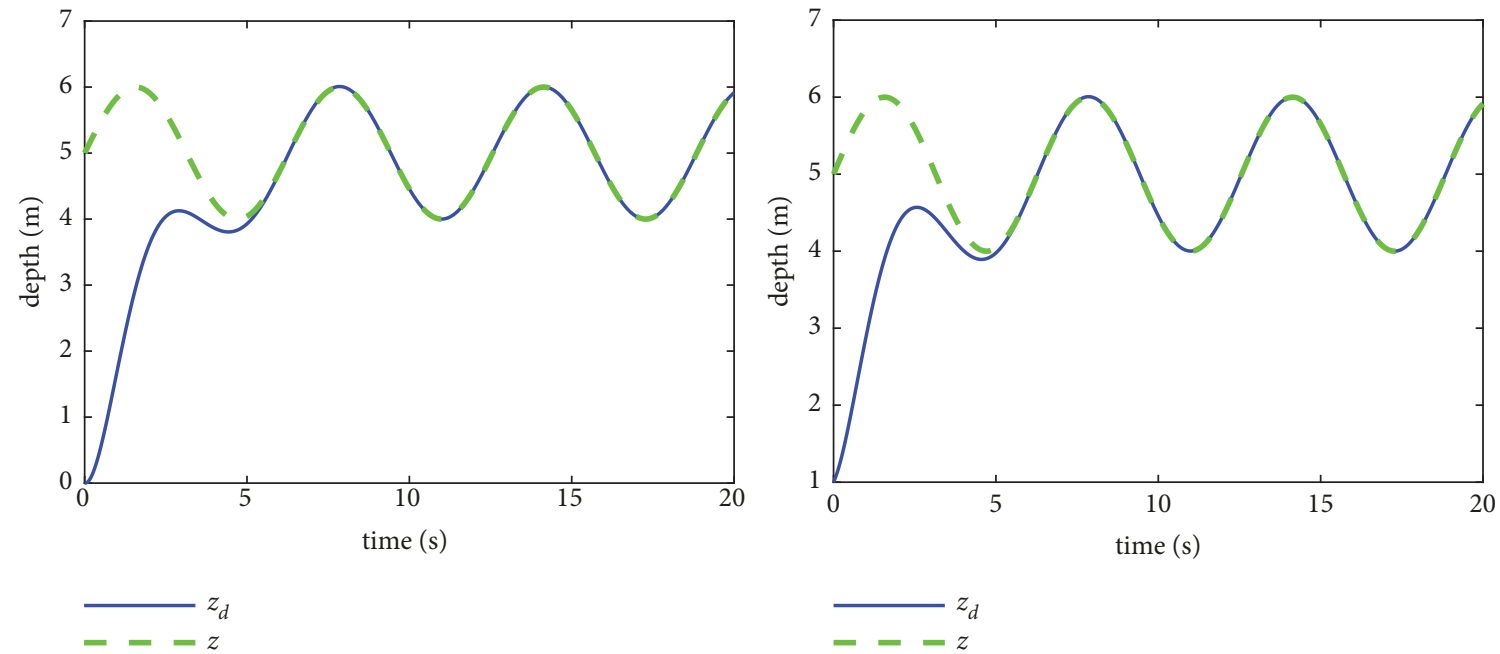

FIGURE 4: The depth tracking signal $z$ and $z_{d}$.
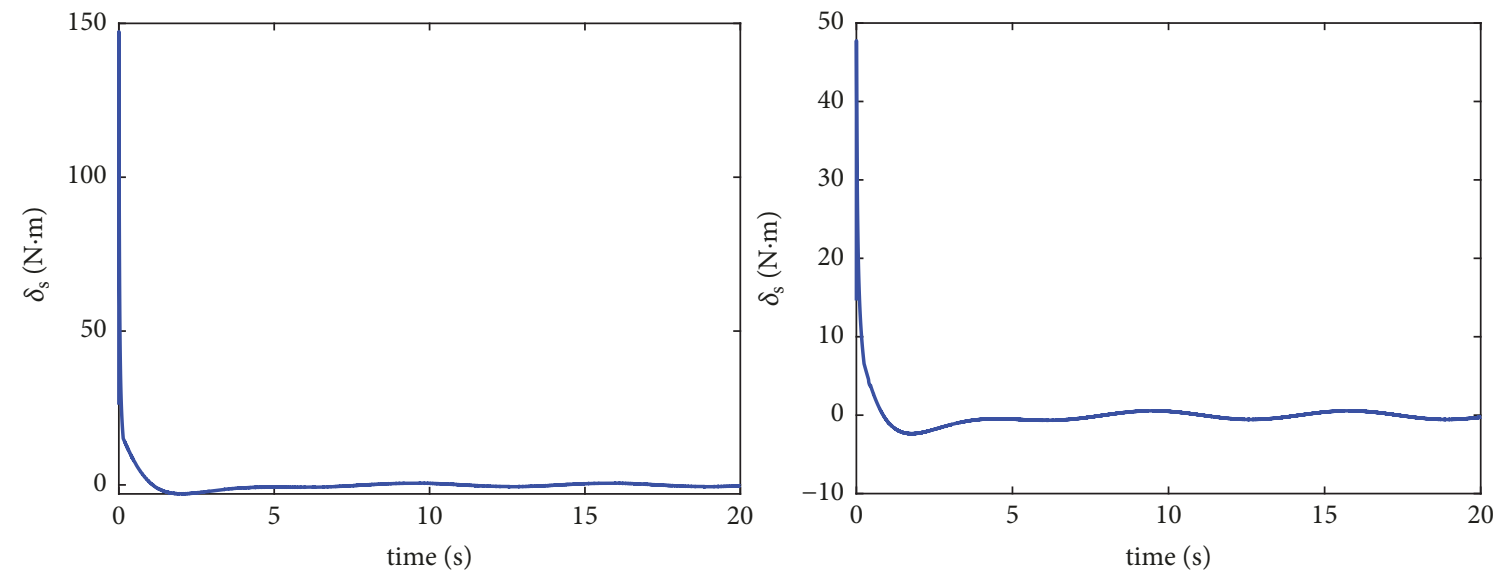

Figure 5: The complete control law $\delta_{s}$ in the entire simulation interval.
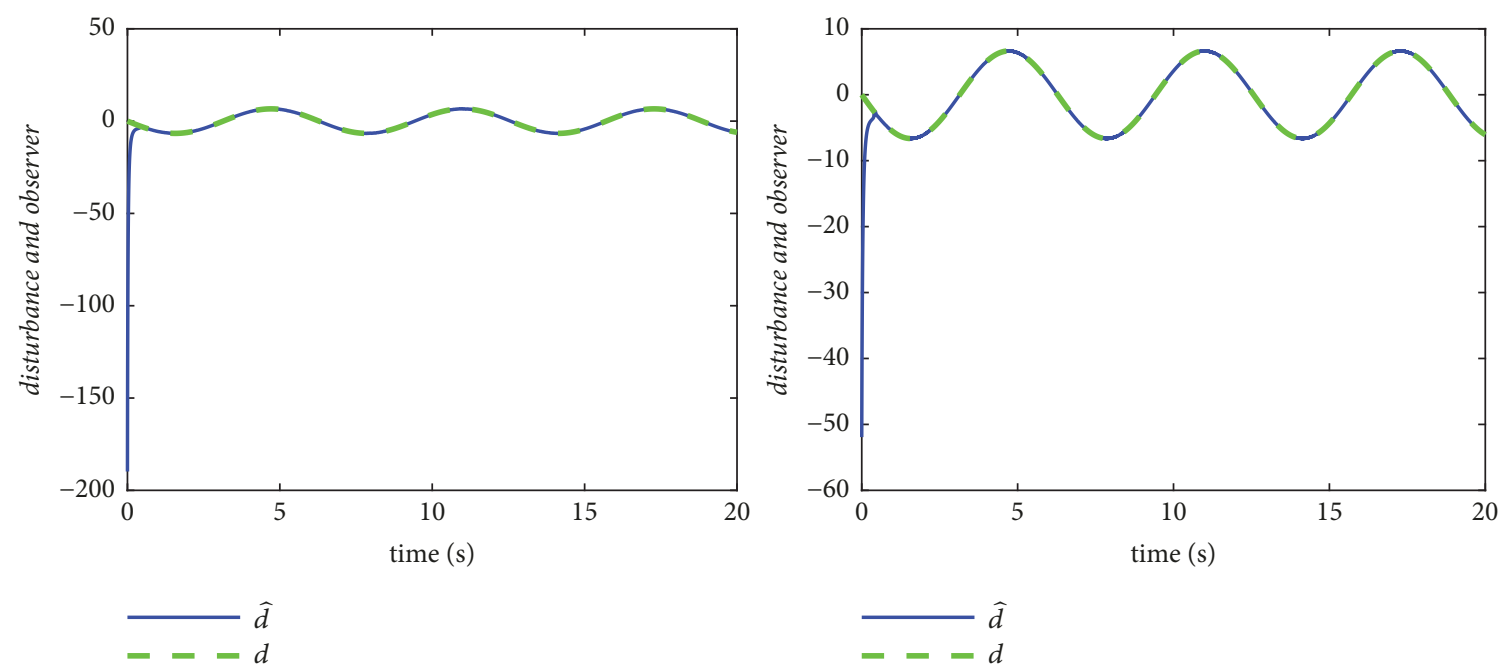

FIgURE 6: The FTSMDO and disturbance in the entire simulation interval. 

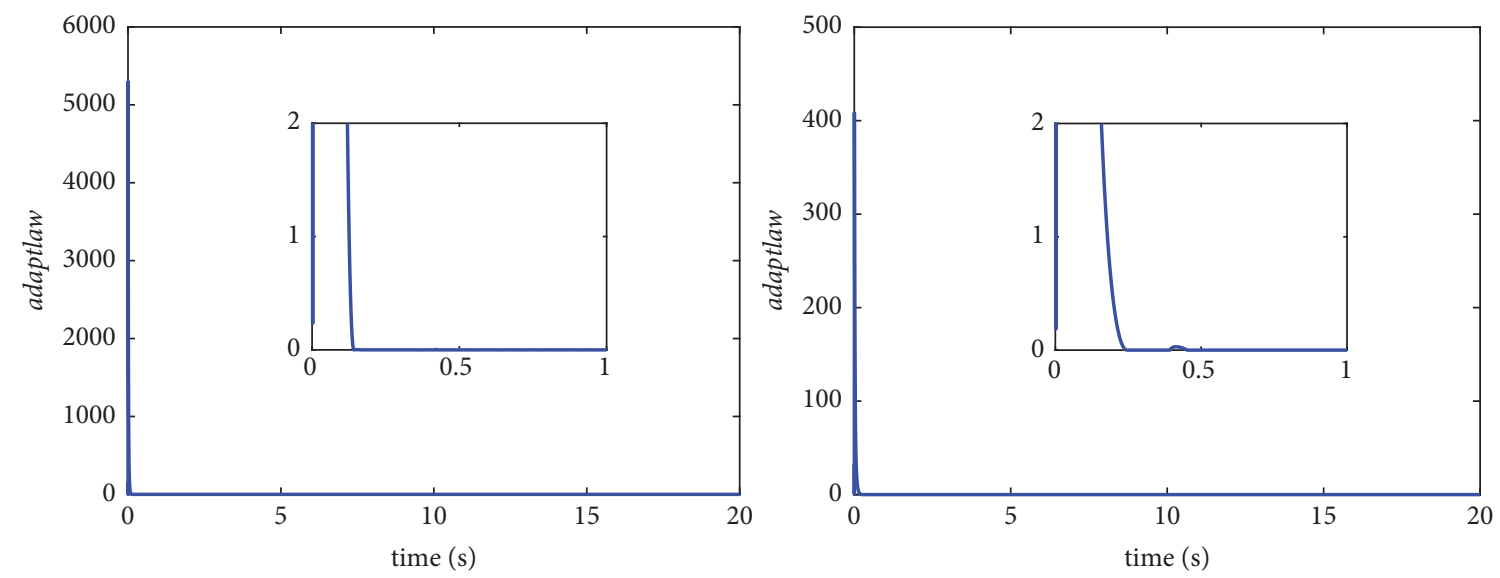

Figure 7: Adapt law.

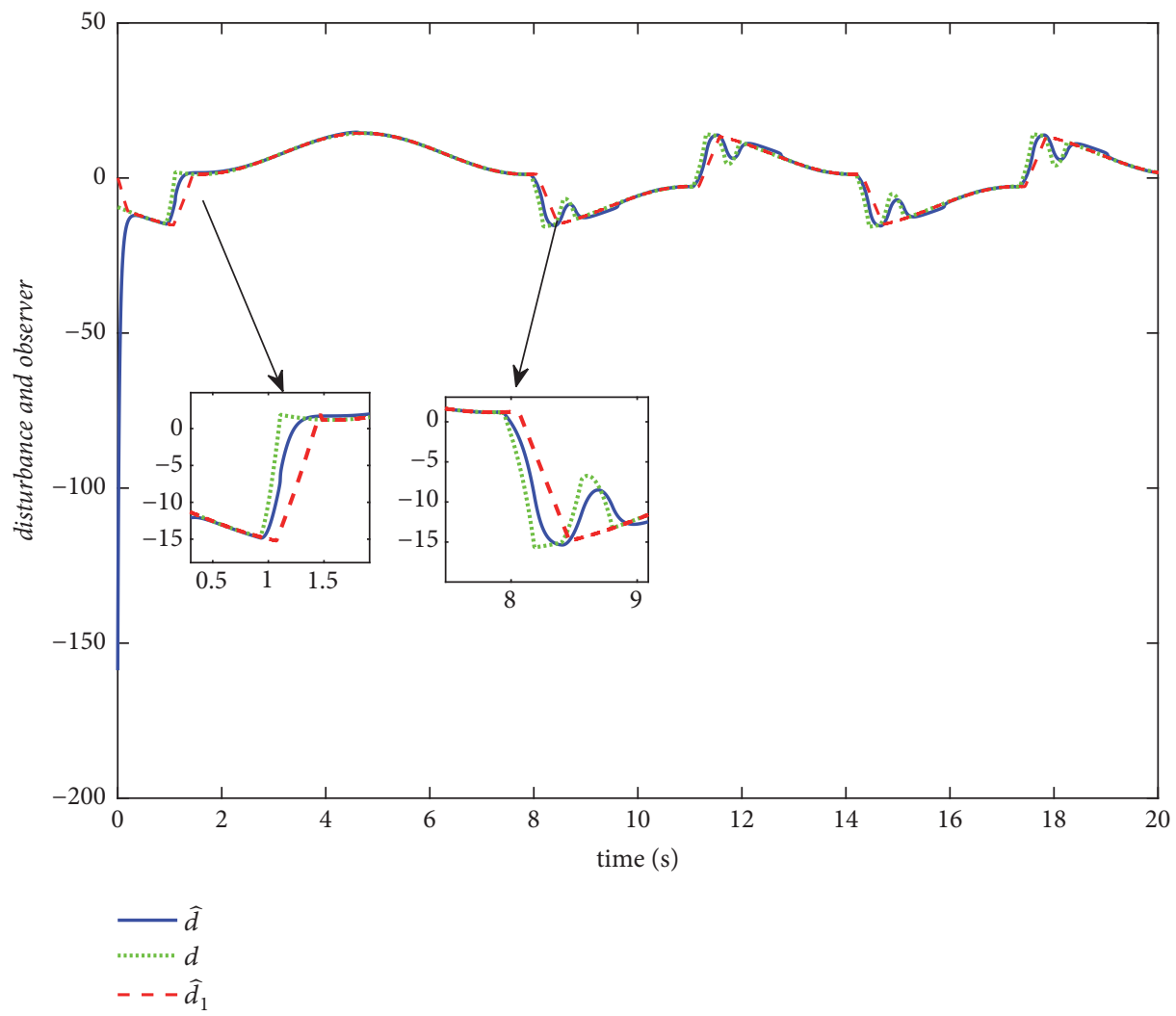

FIGURE 8: Compound disturbance and its estimate in two observers existing dead-zone input.

not following big adjusting. And the convergence time does not exceed the theoretical maximum. It is displayed that the proposed FTSMDO can estimate the disturbance accurately. Simulation results make clear that the designed fixed-time sliding mode controller based on FTSMDO assures fixedtime convergence and better performance properties of the whole system. The control input response curve is smooth and less chattering. At the same time, the proposed method can guarantee the underwater robot's track of the reference trajectory in a fixed-time.
The dead-zone input parameters are set to $b_{l}=-5, b_{r}=$ $6, m=1$. In order to demonstrate the validity and efficiency of the proposed FTSMDO, we conduct the simulation in compares with exact uniform robust disturbance observer in literature [27], defined as $\widehat{d}_{1}$; the controller adopts the highorder finite-time sliding mode method proposed in [37]. The proposed FTSMDO by us is defined as $\widehat{d}$, and the simulation results subject to compound disturbance $d, \widehat{d}$, and $\widehat{d}_{1}$ are depicted respectively in Figure 8. The control input $v$ and $\delta_{s}$ are given, respectively, in two methods in Figure 9. In 

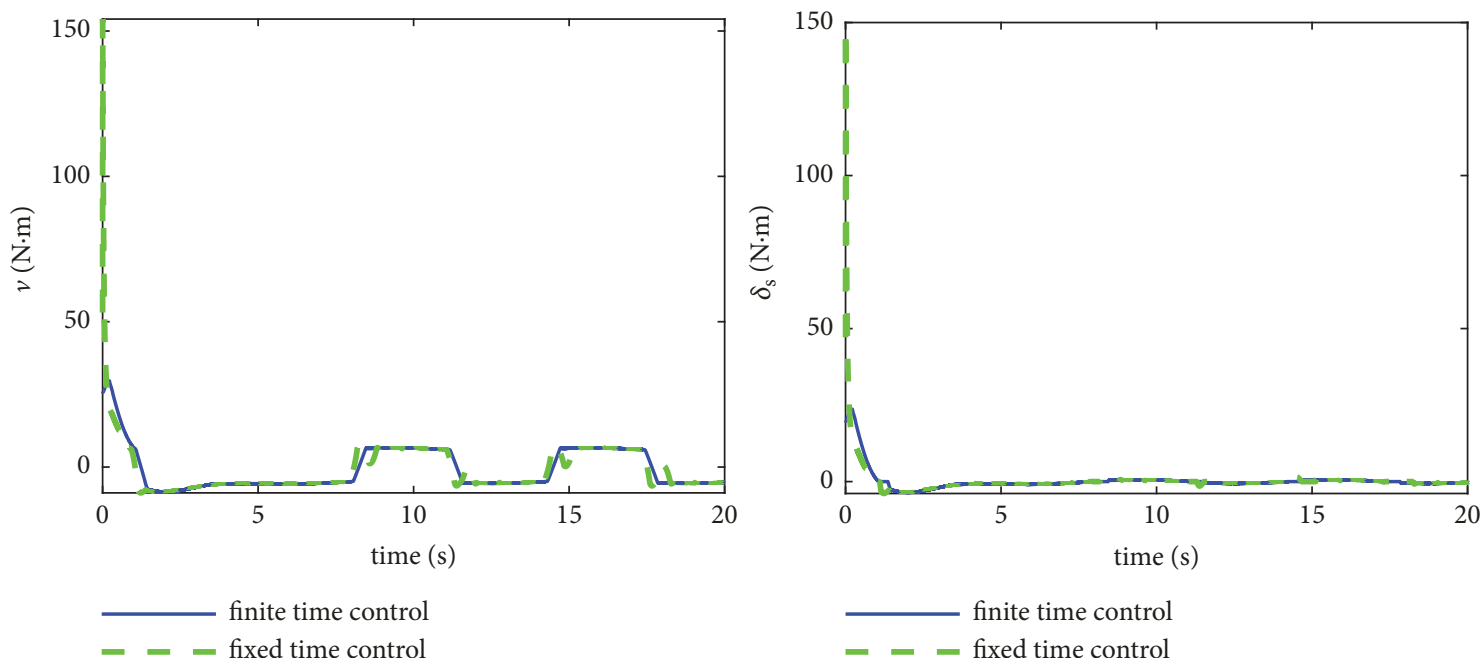

FIGURE 9: The control input $v$ and $\delta_{s}$ for the case of existing dead-zone input in two methods for comparisons.
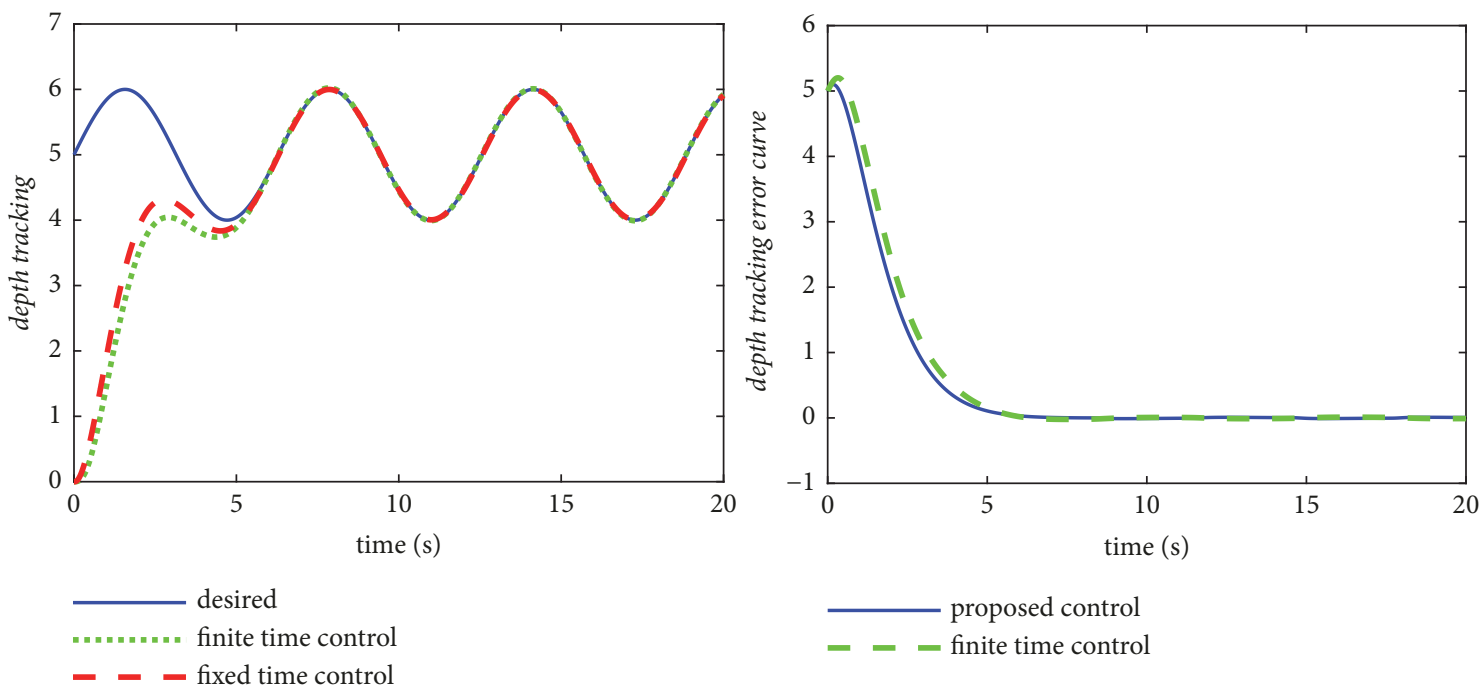

FIGURE 10: The depth tracking trajectory curve and its error cure for the case of existing dead-zone input.

the end, the depth tracking cure and its error are depicted in Figure 10. Figures 8 and 10 show a rapid response of the system by the proposed method compared to the compared method. We can see that the system tends to be stable rapidly, and the output can track the reference signal less time by our presented fixed-time method than the finite-time control approach.

\section{Conclusions}

In this paper, the fixed-time trajectory tracking control problem for high-order integrator system subjects to input dead-zone and external time-varying disturbances is researched. The comprehensive design of FTSMDO and the sliding mode fixed-time approach is studied. Meanwhile, an adapt law is designed for the case where the derivative of interference term is unknown but bounded. The adaptive sliding mode fixed-time method based on FTSMDO is proposed for high-order dynamic systems to guarantee fixed-time stability. And the convergence time of the proposed method for the system is estimated unrelated to the initial states. In the last, the designed control algorithm is employed for a vertical plane of AUV in the presence of input dead-zone and external unknown time-varying disturbance. Conducted numerical simulations confirmed the validity and effectiveness of the theoretical results.

\section{Data Availability}

The data used to support the findings of this study are available from the corresponding author upon request.

\section{Conflicts of Interest}

The authors declare that they have no conflicts of interest. 


\section{Acknowledgments}

This work is supported by the Hebei Province under Grant F2016203496.

\section{References}

[1] H. Du, S. Li, and C. Qian, "Finite-time attitude tracking control of spacecraft with application to attitude synchronization," IEEE Transactions on Automatic Control, vol. 56, no. 11, pp. 2711-2717, 2011.

[2] M. Basin, Y. Shtessel, and F. Aldukali, "Continuous finiteand fixed-time high-order regulators," Journal of The Franklin Institute, vol. 353, no. 18, pp. 5001-5012, 2016.

[3] B. Tian, Z. Zuo, X. Yan, and H. Wang, "A fixed-time output feedback control scheme for double integrator systems," Automatica, vol. 80, pp. 17-24, 2017.

[4] J. P. Mishra, X. Yu, and M. Jalili, "Arbitrary-order continuous finite-time sliding mode controller for fixed-time convergence," IEEE Transactions on Circuits \& Systems II, pp. 1-1, 2018.

[5] Z. Zuo, "Non-singular fixed-time terminal sliding mode control of non-linear systems," IET Control Theory \& Applications, vol. 9, no. 4, pp. 545-552, 2015.

[6] L. Zhang, C. Wei, L. Jing, and N. Cui, "Fixed-time sliding mode attitude tracking control for a submarine-launched missile with multiple disturbances," Nonlinear Dynamics, vol. 93, no. 4, pp. 2543-2563, 2018.

[7] Z. Zhang, X. Liu, Y. Liu, C. Lin, and B. Chen, "Fixed-time almost disturbance decoupling of nonlinear time-varying systems with multiple disturbances and dead-zone input," Information Sciences, vol. 450, pp. 267-283, 2018.

[8] J. Huang and Z. Zhang, "Nonlinear feedback design for fixedtime tracking of a class of nonlinear systems," International Journal of Computer Mathematics, vol. 94, no. 7, pp. 1349-1362, 2016.

[9] H. Wang, B. Su, Y. Wang, and J. Gao, "Distributed robust fixed-time consensus for nonlinear and disturbed multiagent systems," IEEE Transactions on Systems, Man, and Cybernetics: Systems, pp. 1-10, 2016.

[10] Z. Zuo, onsingular Fixed-Time Consensus Tracking for SecondOrder Multi-Agent Networks, Pergamon Press, 2015.

[11] Z. Zuo, B. Tian, M. Defoort, and Z. Ding, "Fixed-time consensus tracking for multiagent systems with high-order integrator dynamics," IEEE Transactions on Automatic Control, p. 1, 2017.

[12] J. Yu, M. Chen, and C. S. Jiang, "Adaptive sliding mode control for nonlinear uncertain systems based on disturbance observer," Control Theory \& Applications, 2014.

[13] Z. Qiang, G. Z. Yuan, and D. Z. Xu, "An adaptive second order terminal sliding mode control for a class of uncertain nonlinear systems using disturbance observer," Control Theory and Application, pp. 32-34, 2017.

[14] Y.-W. Liao, S. Pan, F. Borrelli, and J. K. Hedrick, "Adaptive sliding mode control without knowledge of uncertainty bounds," in Proceedings of the 2018 Annual American Control Conference, ACC 2018, pp. 905-911, USA, June 2018.

[15] M. Hou, Z. Zhang, Z. Deng, and G. Duan, "Global robust finite-time stabilisation of unknown pure-feedback systems with input dead-zone non-linearity," IET Control Theory \& Applications, vol. 10, no. 2, pp. 234-243, 2016.

[16] C. Hua, Y. Li, and X. Guan, "Finite/Fixed-Time stabilization for nonlinear interconnected systems with dead-zone input," IEEE
Transactions on Automatic Control, vol. 62, no. 5, pp. 2554-2560, 2017.

[17] G. Z. Cui, S. Y. Xu, Q. Ma, Y. M. Li, and Z. Q. Zhang, "Prescribed performance distributed consensus control for nonlinear multiagent systems with unknown dead-zone input," International Journal of Control, vol. 91, no. 5, 2018.

[18] W.-H. Chen, D. J. Ballance, P. J. Gawthrop, and J. O’Reilly, “A nonlinear disturbance observer for robotic manipulators," IEEE Transactions on Industrial Electronics, vol. 47, no. 4, pp. 932-938, 2000.

[19] S. Mohammed, W. Huo, J. Huang, H. Rifaï, and Y. Amirat, "Nonlinear disturbance observer based sliding mode control of a human-driven knee joint orthosis," Robotics and Autonomous Systems, vol. 75, pp. 41-49, 2016.

[20] E. Sariyildiz, H. Yu, K. Yu, and K. Ohnishi, "A nonlinear stability analysis for the robust position control problem of robot manipulators via disturbance observer," in Proceedings of the 2015 IEEE International Conference on Mechatronics, ICM 2015, pp. 28-33, Japan, March 2015.

[21] S. D. Nguyen, S.-B. Choi, and Q. H. Nguyen, "A new fuzzydisturbance observer-enhanced sliding controller for vibration control of a train-car suspension with magneto-rheological dampers," Mechanical Systems and Signal Processing, vol. 105, pp. 447-466, 2018.

[22] C. E. Hall and Y. B. Shtessel, "Sliding mode disturbance observer-based control for a reusable launch vehicle," Journal of Guidance, Control, and Dynamics, vol. 29, no. 6, pp. 1315-1328, 2006.

[23] M. Chen, S.-d. Chen, and Q.-x. Wu, "Sliding mode disturbance observer based adaptive control for uncertain MIMO nonlinear systems with dead-zone," International Journal of Adaptive Control and Signal Processing, vol. 31, no. 7, pp. 1003-1018, 2017.

[24] L. Zhu, C. Jiang, and C. Zhang, "Adaptive trajectorylinearization control for aerospace vehicle based on RBFNN disturbance observer," Acta Aeronautica Et Astronaut Ica Sinica, vol. 28, no. 3, pp. 673-677, 2007 (Chinese).

[25] S. D. Nguyen, H. D. Vo, and T. Seo, "Nonlinear adaptive control based on fuzzy sliding mode technique and fuzzy-based compensator," ISA Transactions ${ }^{\circledR}$, vol. 70, pp. 309-321, 2017.

[26] X.-W. Bu, X.-Y. Wu, Y.-X. Chen, and R.-Y. Bai, "Nonlineardisturbance-observer-based sliding mode backstepping control of hypersonic vehicles," Kongzhi Lilun Yu Yingyong/Control Theory and Applications, vol. 31, no. 11, pp. 1473-1479, 2014.

[27] E. Cruz-Zavala, J. A. Moreno, and L. M. Fridman, "Uniform robust exact differentiator," IEEE Transactions on Automatic Control, vol. 56, no. 11, pp. 102-107, 2011.

[28] J. Yang, S. Li, J. Su, and X. Yu, "Continuous nonsingular terminal sliding mode control for systems with mismatched disturbances," Automatica, vol. 49, no. 7, pp. 2287-2291, 2013.

[29] J. Li, Y. Yang, C. Hua, and X. Guan, "Fixed-time backstepping control design for high-order strict-feedback non-linear systems via terminal sliding mode," IET Control Theory \& Applications, vol. 11, no. 8, pp. 1184-1193, 2017.

[30] J. Fei and Z. Feng, "Adaptive fuzzy super-twisting sliding mode control for microgyroscope," Complexity, vol. 2019, no. 6, pp. 113, 2019.

[31] G. Xia, C. Pang, and J. Xue, "Fuzzy neural network-based robust adaptive control for dynamic positioning of underwater vehicles with input dead-zone," Journal of Intelligent \& Fuzzy Systems: Applications in Engineering and Technology, vol. 29, no. 6, pp. 2585-2595, 2015. 
[32] J. Ni, L. Liu, M. Chen, and C. Liu, "Fixed-time disturbance observer design for Brunovsky system," IEEE Transactions on Circuits and Systems II: Express Briefs, 2017.

[33] M. Basin, C. B. Panathula, and Y. Shtessel, "Continuous secondorder sliding mode control: Convergence time estimation," in Proceedings of the 54th IEEE Conference on Decision and Control, CDC 2015, pp. 5408-5413, Japan, December 2015.

[34] M. Basin, P. Yu, and Y. Shtessel, "Finite- and fixed-time differentiators utilising HOSM techniques," IET Control Theory \& Applications, vol. 11, no. 8, pp. 1144-1152, 2017.

[35] C. Wei, W. Yanhui, and Z. Jianhui, "Back-stepping control of underactuated AUV's depth based on nonlinear disturbance observer," in Proceedings of the 2015 34th Chinese Control Conference (CCC), pp. 6061-6065, Hangzhou, China, July 2015.

[36] X. Yao, G. Yang, and Y. Peng, "Nonlinear reduced-order observer-based predictive control for diving of an autonomous underwater vehicle," Discrete Dynamics in Nature and Society, vol. 2017, Article ID 4394571, 15 pages, 2017.

[37] Y. Feng, F. Han, and X. Yu, "Chattering free full-order slidingmode control," Automatica, vol. 50, no. 4, pp. 1310-1314, 2014. 


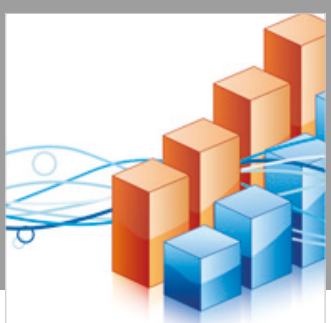

Advances in

Operations Research

\section{-n-m}
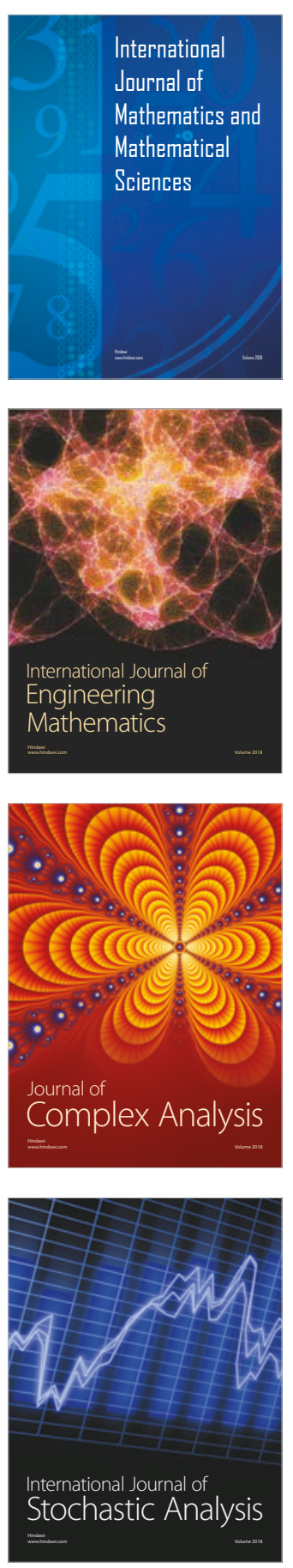
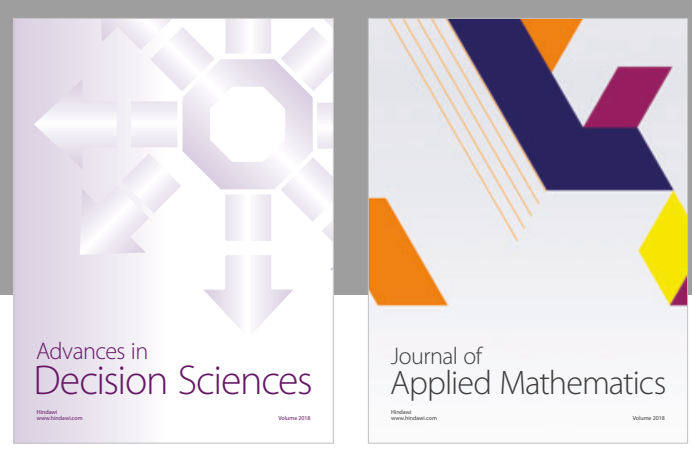

Journal of

Applied Mathematics
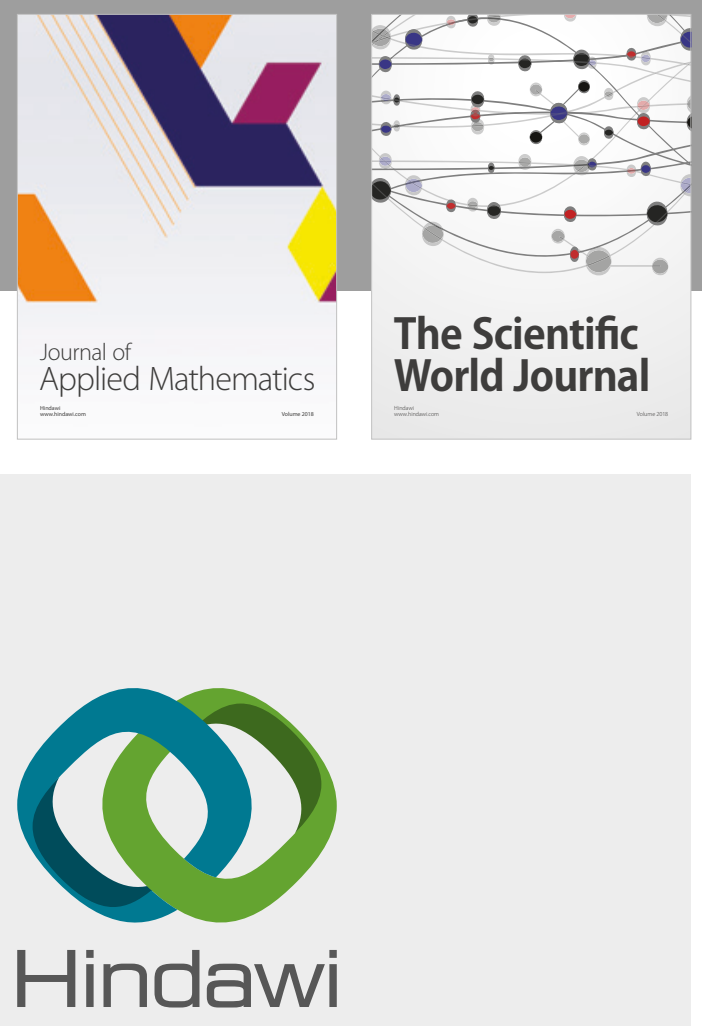

Submit your manuscripts at

www.hindawi.com

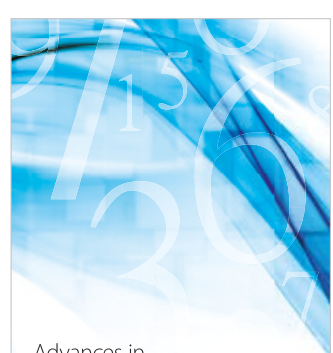

Advances in
Numerical Analysis
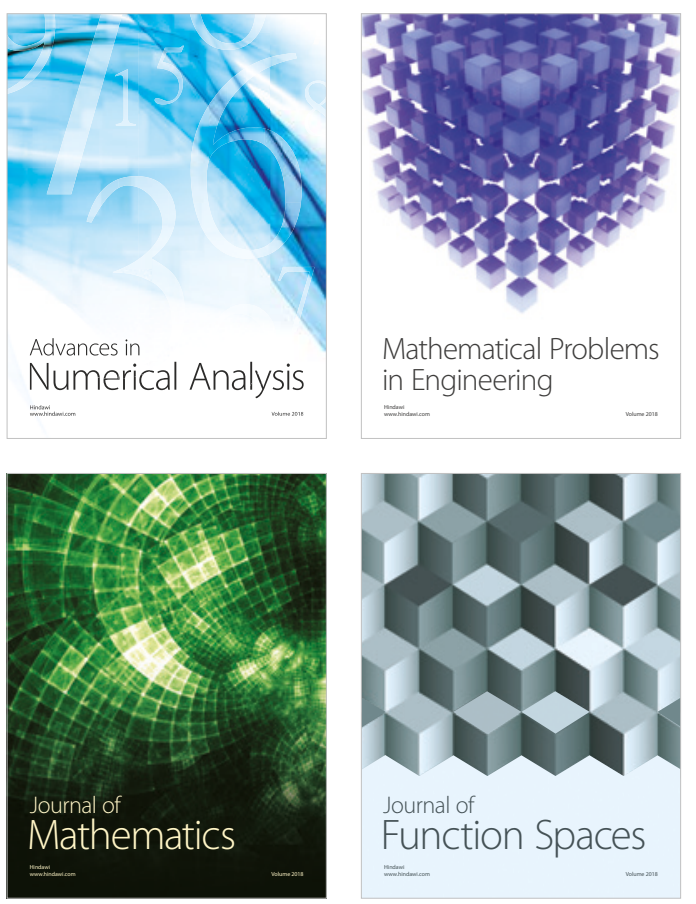

Mathematical Problems in Engineering

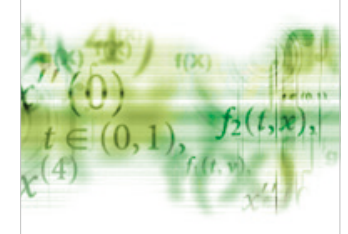

International Journal of

Differential Equations

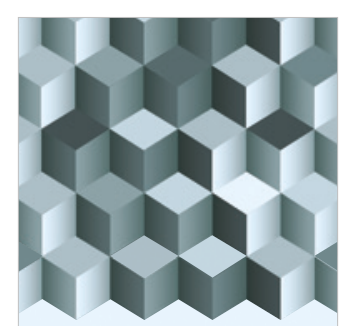

Journal of

Function Spaces
The Scientific

World Journal

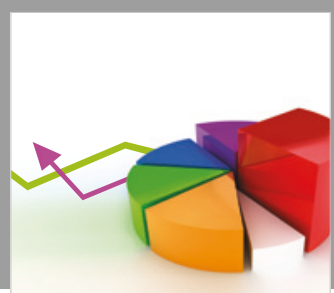

Journal of

Probability and Statistics
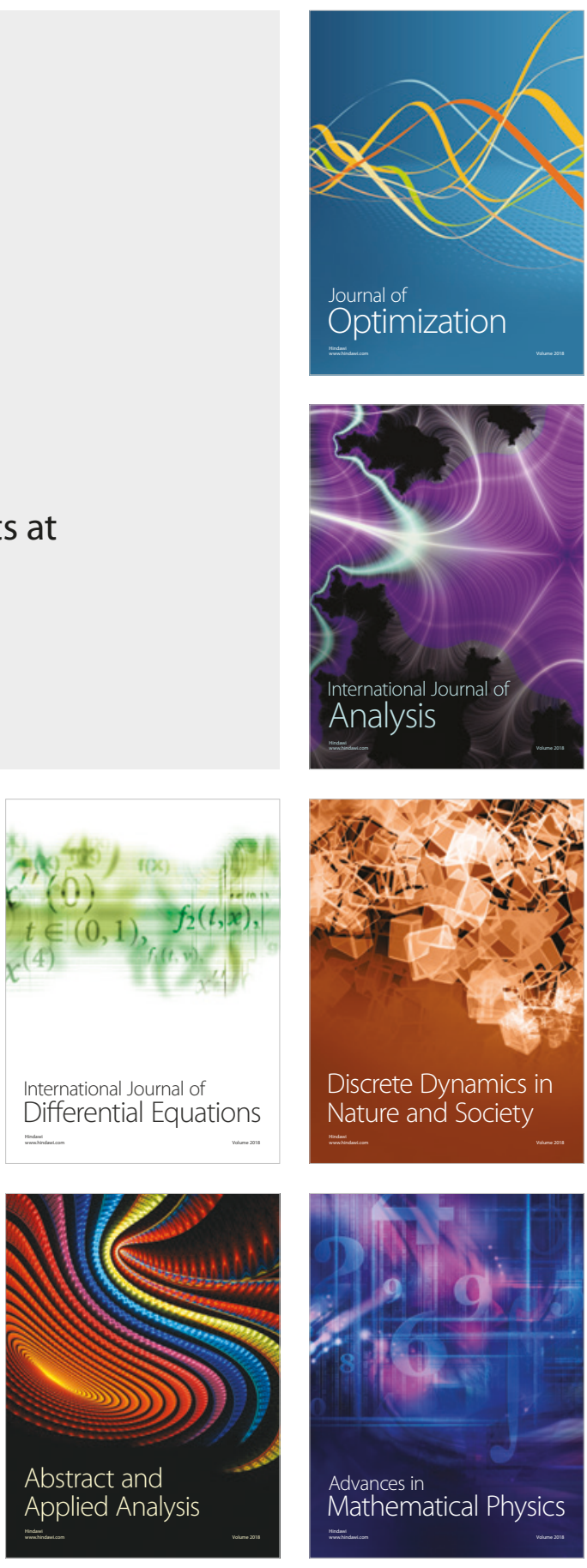